Enfermagem Brasil 2018;17(1):43-8

\title{
ARTIGO ORIGINAL \\ Uso de álcool, tabaco e outras drogas por estudantes do ensino médio integrado de uma escola da região oeste catarinense
}

\author{
Alessandra Domingues Malheiro, M.Sc. ${ }^{*}$, Grazieli Ferreira da Rosa** \\ ${ }^{*}$ Enfermeira do Trabalho e Saúde Coletiva, Mestre em Ciências Sociais pela PUC/RS, \\ Professora de Saúde e Segurança do Trabalho no IFC Videira, ${ }^{* * E n f e r m e i r a ~ d o ~ T r a b a l h o, ~}$ \\ Enfermeira do IFC Videira
}

Recebido em 15 de julho de 2017; aceito em 3 de novembro de 2017.

Endereço para correspondência: Alessandra Domingues Malheiro, Rua Santa Catarina, 18/8, 89560-000 Videira SC, E-mail: alessandra.malheiro@ifc.edu.br; Grazieli Ferreira da Rosa: grazieli.rosa@ifc.edu.br

\begin{abstract}
Resumo
Objetivo: Realizar um levantamento sobre uso de drogas entre estudantes do ensino médio integrado de uma escola da região oeste catarinense. Métodos: Todos os alunos do ensino médio integrado da escola foram convidados a participar do estudo. Aplicou-se questionário não identificado aos estudantes que aceitaram participar. Resultados: Participaram do estudo 246 alunos. A maior parte da amostra foi do sexo feminino $(49,6 \%)$, no entanto os meninos apresentaram uma maior experimentação de drogas quando comparados com as meninas. A maior parte dos entrevistados $(58,6 \%)$ já havia utilizado algum tipo de droga. As drogas mais utilizadas foram: álcool e narguilé. Conclusão: Devido ao elevado número de jovens que experimentam drogas é extremamente importante ações de prevenção em saúde.
\end{abstract}

Palavras-chave: adolescente, drogas ilícitas, tabaco, consumo de álcool por menores.

\begin{abstract}
Use of alcohol, tobacco and other drugs by high school students in a school in the west region of Santa Catarina

Objective: To conduct a survey on drug use among high school students integrated in a school in the western region of Santa Catarina. Methods: All high school integrated students were invited to participate in the study. An unidentified questionnaire was applied to students who agreed to participate. Results: 246 students participated in the study. The majority of the sample was female $(49.6 \%)$, however, the boys presented a greater experimentation of drugs when compared with the girls. Most of the interviewees (58.6\%) had already used some type of drug. The most commonly used drugs were alcohol and narghile. Conclusion: Due to the high number of young people who experience drugs, health prevention actions are extremely important.
\end{abstract}

Key-words: adolescent, street drugs, tobacco, underage drinking.

\section{Resumen}

Uso de alcohol, tabaco y otras drogas por estudiantes de la enseñanza media integrada de una escuela de la región oeste catarinense

Objetivo: Realizar un levantamiento sobre el uso de drogas entre estudiantes de enseñanza media integrada de una escuela de la región oeste catarinense. Métodos: Todos los alumnos de la escuela secundaria fueron invitados a participar en el estudio. Se aplicó un cuestionario no identificado a los estudiantes que aceptaron participar. Resultados: Participaron del estudio 246 alumnos. La mayor parte de la muestra fue del sexo femenino (49,6\%), sin embargo, los niños presentaron una mayor experimentación de drogas cuando comparados con las niñas. La mayoría de los encuestados $(58,6 \%)$ ya habían utilizado algún tipo de droga. Las drogas más utilizadas fueron: alcohol y narguile. Conclusión: Debido al elevado número de jóvenes que experimentan drogas es extremadamente importante acciones de prevención en salud.

Palabras-clave: adolescente, drogas ilícitas, tabaco, consumo de alcohol por menores. 
A adolescência é uma construção sócio-histórica cujas manifestações são fortemente influenciadas pelos fatores socioeconômicos, políticos e culturais do ambiente onde 0 adolescente vive [1].

Faz parte da adolescência a busca por novas experiências e sensações, incluindo a curiosidade pelo uso das drogas, tanto as lícitas quanto as ilegais [2]. Além disso, é uma fase vulnerável à aquisição de hábitos, os quais podem se tornar duradouros ao longo da vida [3], levando a risco aumentado para desenvolver patologias, como câncer, hipertensão arterial, doenças cardíacas, entre outras. O abuso de drogas também está relacionado com a violência urbana, que muito tem vitimado jovens nos grandes centros do Brasil.

A etiologia do uso de substâncias pelos adolescentes é desconhecida. O problema, multifatorial, engloba fatores genéticos ou familiares, influências dos companheiros, traços individuais, influências sociais e psicológicas [4]. Ainda importante ressaltar a influência das mídias sociais no desenvolvimento de hábitos e costumes entre adolescentes, nos dias atuais.

A escola tem papel importante na formação de diversos valores e comportamentos. Trata-se de um ambiente propício para adquirir novos conhecimentos e habilidades, por meio de troca de informações e experiências e contato com diferentes culturas [5]. A escola consegue atingir não apenas o estudante, mas também a família e a comunidade, sendo desta forma um ambiente de grande valia para o desenvolvimento de ações de prevenção ao uso de drogas entre jovens.

Assim, este estudo teve como objetivo realizar um levantamento sobre uso de drogas entre estudantes do ensino médio integrado de uma escola técnica da região oeste catarinense.

\section{Material e métodos}

Trata-se de um estudo transversal, descritivo de abordagem quantitativa. A coleta de dados ocorreu no período entre fevereiro e junho de 2015, em uma escola pública da rede federal de ensino, localizada na região oeste catarinense.

A escolha do local para a realização da pesquisa deu-se pela necessidade de conhecer os estilos de vida dos jovens da escola, com o objetivo de desenvolver ações de saúde a partir das demandas levantadas. Esta pesquisa faz parte de um projeto maior em que outro instrumento de coleta de dados avaliou questões referentes à alimentação, peso, estatura, atividades de lazer, patologias prévias.

$\mathrm{Na}$ escola em que foi realizada a pesquisa, no ano de 2015, estudavam aproximadamente 1.000 pessoas, distribuídas em cursos de ensino médio integrado, cursos técnicos subsequentes e cursos de graduação. A amostra deste estudo se constituiu dos estudantes do ensino médio integrado (primeiro, segundo e terceiro anos) dos cursos de informática, eletrotécnica e agropecuária. Excluiu-se do estudo aqueles estudantes que não aceitaram participar, bem como aqueles ausentes da escola no momento da coleta dos dados.

Todos os alunos do ensino médio integrado foram convidados a participar da pesquisa, esclarecidos acerca dos seus objetivos, riscos e benefícios, e a participação foi voluntária. Os estudantes que aceitaram participar assinaram o termo de assentimento, assim como seus responsáveis (também após os mesmos esclarecimentos), assinaram o termo de consentimento. O presente trabalho faz parte de um projeto de pesquisa maior, foi cadastrado na plataforma Brasil e encaminhado ao Comitê de Ética do Centro Educacional Luterano Bom e foi autorizada sua realização sob o parecer 1.114.078.

Dos 357 alunos matriculados no ensino médio integrado em fevereiro de 2015, participaram 246 para os quais foi aplicado questionário em que o participante não se identificava. $O$ instrumento de coleta de dados solicitava apenas idade, sexo e uso de substâncias químicas. Os questionários foram aplicados em sala de aula, com apoio dos professores pesquisadores. Após a coleta dos dados, foi realizada a análise de cada questionário, e os dados obtidos foram compilados em tabelas (em números absolutos e em forma de percentual).

Todos os alunos do ensino médio integrado da escola pesquisada apresentavam entre 14 e 18 anos no momento da pesquisa. Dos 246 estudantes que aceitaram participar do estudo, 
122 (49.6\%) eram do sexo feminino, $118(48 \%)$ do sexo masculino, e 6 ( 2,4\%) não informaram o sexo.

A tabela I foi desenvolvida com o objetivo de demonstrar as respostas obtidas, por sexo, a partir da pergunta: Você já utilizou algum tipo de droga (lícita ou ilícita)? A maior parte dos participantes $(58,6 \%)$ desta pesquisa já experimentou algum tipo de droga.

Tabela I - Uso de drogas lícitas e ilícitas de acordo com o sexo. Fevereiro a junho de 2015. Região Oeste Catarinense, SC.

\begin{tabular}{lllll}
\hline Sexo & Já usou (n) & $\%$ & Nunca usou (n) & $\%$ \\
\hline Feminino & 63 & 25,7 & 59 & 24,0 \\
Masculino & 75 & 30,5 & 43 & 17,4 \\
Não informou & 6 & 2,4 & 0 & 0,0 \\
Total & 144 & 58,6 & 102 & 41,4 \\
\hline
\end{tabular}

Dos $144(58,6 \%)$ que responderam já ter utilizado algum tipo de droga, 131 (91\%) relataram já ter usado álcool alguma vez na vida, $55(38,1 \%)$ referiram o uso de narguilé por pelo menos uma vez. O cigarro de tabaco aparece em terceiro lugar quando se trata de droga já experimentada pelos jovens entrevistados, relatada por $27(16,6 \%)$ dos que responderam já ter usado algum tipo de droga. Com relação às drogas ilegais, a maconha foi apontada por 21 $(14,58 \%)$ dos que responderam sim para o questionamento sobre uso de drogas, e substâncias como cocaína, LSD, heroína e outras não especificadas também foram apontadas pelos jovens, conforme a tabela II. Alguns jovens apontaram já ter usado mais de um tipo de droga.

Tabela II - Drogas já utilizadas pelos adolescentes entrevistados. Fevereiro e junho de 2015. Região Oeste Catarinense, SC.

\begin{tabular}{lll}
\hline Droga & Quantos Informaram (N) & $\%$ \\
\hline Álcool & 131 & 53,2 \\
Narguilé & 55 & 22,3 \\
Cigarro (tabaco) & 27 & 11,0 \\
Maconha & 21 & 8,5 \\
Cocaína & 6 & 2,4 \\
LSD & 6 & 2,4 \\
Heroína & 3 & 1,21 \\
Não especificado & 3 & 1,21 \\
\hline
\end{tabular}

Discussão

O uso de drogas, lícitas ou ilícitas está entre os principais problemas a serem prevenidos na adolescência. A amostra deste estudo constituiu-se de jovens de 14 a 18 anos, estudantes de uma escola pública de ensino médio integrado do oeste catarinense, na qual estes alunos estudam cinco dias da semana, em turno integral, e no final dos 3 anos o aluno recebe um diploma de curso técnico.

Dos 246 alunos que responderam o questionário, 144 (58,6\%) já haviam utilizado algum tipo de droga. A amostra constituiu-se de um maior número de meninas: $122(49,6 \%)$ do que meninos: $118(48 \%)$, e ainda $6(2,4 \%)$ não informaram o sexo. O maior número de jovens que afirmou já ter experimentado algum tipo de droga foi do sexo masculino: 75 contra 63 do sexo feminino.

No que se refere aos tipos de drogas experimentadas pelos pesquisados, 131 (53,2\%) já haviam usado álcool pelo menos uma vez na vida. Este dado é semelhante ao encontrado no PeNSE (Pesquisa Nacional de Saúde do Escolar) 2015 [6] que mostrou que a experimentação de bebida alcoólica por escolares do 9o ano foi de 55,5\% e o índice aumenta quando se trata da faixa etária de 16 a 17 anos: $73 \%$ já experimentaram uma dose de bebida alcoólica [6]. Em outro estudo realizado em município do Sul do Brasil [7] com adolescentes de 12 a 18 anos, o índice de uso de bebida alcoólica pelo menos uma vez na vida foi de $60,7 \%$. 0 PeNSE 2015 aponta ainda que a região Sul do Brasil concentra o maior percentual de escolares com consumo atual de bebidas alcoólicas [6]. 
O álcool pode agir como estimulante em uma primeira fase e deixa a pessoa desinibida e eufórica, mas à medida que as doses aumentam, começam a surgir os efeitos depressores, que levam a diminuição da coordenação motora, dos reflexos e sono. $\mathrm{O}$ uso prolongado pode causar alcoolismo, cirrose e câncer no fígado. É importante ressaltar que o consumo de álcool provoca agressividade e pode trazer prejuízos ao corpo do adolescente, pode ainda aumentar a vulnerabilidade para infecções sexualmente transmissíveis, pela ausência do uso do preservativo nas relações sexuais. A violência e acidentes também estão relacionados ao consumo de álcool [2]. Apesar de proibida a venda do álcool para menores de 18 anos, o adolescente tem acesso a esta droga muitas vezes na sua própria casa, muitos inclusive, convivem com familiares usuários desta substância, que normalmente é aceita em nossa sociedade.

Um dado que chama bastante atenção são os 55 (22,3\%) estudantes que já fizeram uso de narguilé. Apesar de o narguilé ser uma droga lícita que tem na sua composição o tabaco, separamos a mesma do cigarro comum por ter sido a segunda droga mais citada neste estudo, e pelos riscos à saúde pouco discutidos nas campanhas de prevenção, que em geral enfatizam o cigarro, e deixam de lado outras formas de uso do tabaco.

Existem diferentes produtos de tabaco, preparados de formas distintas que podem ser divididos em produtores e não produtores de fumaça quanto a sua utilização. Dentre os que produzem fumaça, além do cigarro, temos charuto, cachimbo e narguilé. $O$ tabaco que não produz fumaça pode ser encontrado em preparações para ser mascado ou para ser absorvido pela mucosa oral ou nasal. Entretanto todas as formas de utilização de tabaco liberam nicotina para o sistema nervoso central com risco para causar dependência, e não existe forma segura de consumo do tabaco, tendo seus usuários risco aumentado para adoecimento e morte prematura por enfermidades relacionadas ao uso do tabaco [8].

Existem sugestões de que o narguilé surgiu na Índia há mais de 400 anos, porém nos últimos anos tem-se observado um renascimento nesta forma de uso do tabaco, principalmente entre os jovens. A composição do tabaco para esta modalidade de consumo não é padronizada e seu conteúdo de nicotina é estimado entre $2 \%$ e $4 \%$ em comparação com $1-3 \%$ do tabaco usado para cigarros, e o monóxido de carbono e outras substâncias químicas está em maior percentual na fumaça do narguilé do que na do cigarro. O fumante de narguilé deve inalar, em uma sessão, a mesma quantidade de fumaça que um fumante de cigarros inalaria se consumisse 100 ou mais cigarros [8]. Estudo realizado com estudantes de medicina mostrou que $47 \%$ dos entrevistados já haviam experimentado narguilé [9].

Quanto ao uso de tabaco na forma de cigarro, 27 (11\%) dos alunos entrevistados, afirmaram já ter utilizado pelo menos uma vez na vida. A experimentação de cigarro, assim como a de álcool também é maior na região sul do Brasil comparado às outras regiões [6]. Estudo nacional demonstrou que para escolares entre 13 e 15 anos o índice de experimentação é de 19\% [6]. A influência da família e dos amigos no consumo do tabaco é bastante relevante [7]. O Brasil é um dos principais produtores e exportadores de tabaco no mundo, e a região Sul do país concentra as maiores indústrias fumageiras [7].

As drogas ilícitas citadas pelos entrevistados deste estudo foram a maconha, a cocaína, o LSD (Dietilamida do ácido lisérgico) e a heroína. A maconha, nome popular da planta Cannabis sativa, já foi utilizada por 21 (8,5\%) entrevistados, índice próximo ao apontado pela pesquisa nacional com os escolares de 16 a 17 anos, que demonstrou que o consumo atual de maconha é de $7,2 \%$ nesta faixa etária [6]. Se fumada em pequenas quantidades, a maconha pode alterar a percepção do indivíduo quanto ao gosto, tato, olfato e tempo. Ao longo do tempo prejudica a memória, diminui os reflexos, pode causar problemas respiratórios e aumenta as chances de desenvolver câncer de pulmão [2].

Tanto a cocaína como o LSD já haviam sido utilizadas por 6 jovens entrevistados cada. A cocaína é uma substância extraída das folhas da coca que provoca nos usuários a sensação de alerta, euforia, autoconfiança, mas pode provocar sensação de perseguição, ansiedade, isolamento, pânico e agressividade. Altera frequência cardíaca, pressão arterial e temperatura corporal [2]. O LSD (dietilamida do ácido lisérgico) é uma droga alucinógena muito potente, utilizada geralmente por via oral. Nas fases de alucinação mais intensas pode ocorrer ansiedade, desorientação e pânico. O LSD é capaz de produzir distorções na percepção do ambiente, pode causar ainda psicose duradoura [10]. A heroína (droga opióide) havia sido utilizada por 3 jovens deste estudo. A Pesquisa Nacional de Saúde do Escolar de 2015 concluiu que $17 \%$ dos jovens brasileiros entre 16 e 17 anos já consumiram drogas ilícitas [6].

O consumo abusivo de drogas é mais um sintoma do que a causa de problemas em nossa sociedade e deve ser tratado tendo em vista a complexidade e magnitude do assunto 
[11]. Os dados encontrados nesta pesquisa, assim como em tantas outras, apontam para um elevado número de jovens que já experimentou drogas lícitas ou ilícitas. O uso de álcool e outras drogas está associado a diversos fatores de risco, acarretando prejuízos à saúde e à vida dos adolescentes. Um estudo com adolescentes brasileiros de ambos os sexos [12] observou associações entre violência física e ser vítima de bullying com o uso de drogas ilícitas e efeito potencializador do consumo de álcool e drogas, e para o sexo masculino, o uso de álcool mostrou associação significante com violência física. Desta forma torna-se urgente a ação das famílias, escolas e sociedade para desenvolver medidas de promoção à saúde e prevenção do uso destas substâncias [3].

Em um estudo [13] em uma comunidade do Rio de Janeiro, foi encontrado que os jovens diferenciavam drogas lícitas de ilícitas, citando os tipos mais usados, no entanto classificavam as lícitas como "melhores" já que disseram que estas não traziam tantos agravos. Estes achados demonstram a banalização do uso de drogas, principalmente daquelas permitidas por lei, bem como o desconhecimento por parte dos jovens dos reais malefícios do consumo destas substâncias para a sua saúde.

Uma das vias mais promissoras para promover a adoção de comportamentos saudáveis e a modificação de condutas prejudiciais à saúde é a educação para a saúde, que deve começar na família e prolongar-se por todas as fases do sistema educativo [14]. A escola é um lócus privilegiado para ações de promoção à saúde, porque permite alcançar os estudantes individual e coletivamente e conta com o apoio dos professores e acesso aos pais e familiares [15]. No entanto a educação em saúde na escola não deve ser tratada como intervenções pontuais, e sim comparecer no currículo da formação de crianças e adolescentes como uma abordagem transversal e interdisciplinar [16].

A partir da análise dos dados desta pesquisa, algumas atividades de prevenção ao uso de drogas já foram realizadas na escola, no entanto ainda é necessário que muito mais seja feito, para isto é importante também que escola, unidade de saúde, associações de bairro, entre outras instituições trabalhem de forma conjunta com o objetivo de combater este problema em nossa sociedade. Ressalta-se, ainda, a necessidade de criação de espaços públicos de lazer voltados aos jovens, com atividades orientadas, como alternativas para preencher o tempo ocioso de forma saudável.

O uso de drogas na adolescência é um problema crescente em todo o mundo, principalmente no Brasil. Este estudo apontou um número elevado de adolescentes do ensino médio de uma escola da região oeste catarinense que já experimentaram drogas, principalmente álcool e narguilé. Esta última droga, apesar de ser uma das formas de uso do tabaco, é pouco tratada nas campanhas de prevenção de drogas que têm focado nos malefícios do cigarro.

A educação para a saúde é uma ferramenta para a prevenção do uso de drogas na adolescência, no entanto as atividades voltadas para a saúde nas escolas não devem ser apenas palestras pontuais, mas sim trabalhos contínuos, desenvolvidos de forma interdisciplinar, envolvendo além da escola, as unidades de saúde, a família e a comunidade do bairro e cidade onde está inserida a escola.

Podemos apontar como limitações deste estudo o fato de não apontar a frequência com que os entrevistados usam ou usaram drogas, apenas identificou-se o fato de já ter usado ou não e quais os tipos. Além disso, não foi possível apontar como se deu o contato com as drogas citadas, isto é, se o uso ocorreu em casa com o consentimento dos pais (principalmente no que diz respeito às drogas lícitas), ou foi oferecido por colegas, amigos. Novos estudos abordando uso de drogas entre adolescentes são importantes a fim de que possam fornecer subsídios para formulação de políticas públicas nesta área.

\section{Referências}

1. Brasil. Ministério da Saúde. Secretaria de Atenção à Saúde. Departamento de Ações Programáticas Estratégicas. Orientações básicas de atenção integral à saúde de adolescentes nas escolas e unidades básicas de saúde. Brasília: MS; 2013.

2. Brasil. Saúde para você: Jovens e Adolescentes: Drogas. [citado 2017 Mar 17]. Disponível em URL: http://www.portalsaude.saude.gov.br. 
3. Malta DC, Mascarenhas MDM, Porto DL, Duarte EA, Sardinha LM, Barreto SM, et al. Prevalência do consumo de álcool e drogas entre adolescentes: análise dos dados da Pesquisa Nacional de Saúde Escolar. Rev Bras Epidemiol 2011;14(1)Supl.:136-46.

4. Passos SRL. Ministério da Saúde. Secretaria de Atenção à Saúde. Departamento de Ações Programáticas Estratégicas. Saúde do Adolescente: competências e habilidades. Abuso e dependência de substâncias psicoativas. Brasília: Ministério da Saúde; 2008.

5. Brasil. Ministério da Saúde. Caderno de Atenção Básica no 24 - Saúde na Escola. Brasília: Ministério da Saúde; 2009.

6. Brasil. Instituto Brasileiro de Geografia e Estatística (IBGE). Pesquisa Nacional de Saúde do Escolar 2015. Rio de Janeiro: IBGE; 2016.

7. Vieira PC, Aerts DRGC, Freddo SL, Bittencourt A, Monteiro L, et al. Uso de álcool, tabaco e outras drogas por adolescentes escolares em município do Sul do Brasil. Cad Saúde Pública 2008;24(11):2487-98.

8. Viegas CAA. Formas não habituais de uso do tabaco. J Bras Pneumol 2008;34(12): 1069-73.

9. Martins SR, Paceli RB, Bussacos MA, Fernandes FLA, Prado GF, Lombardi SEM, et al. Experimentação de e conhecimento sobre narguilé entre estudantes de medicina de uma importante universidade do Brasil. J Bras Pneumol 2014;40(2).

10. Gil GF, Gimenez JV, Sauez CB. Drogas alucinógenas e sua detecção laboratorial. ACIS 2014;2(3). [citado 2017 Mar 18]. Disponível em URL: http://revistaseletronicas.fmu.br.

11. Guimarães JL, Godinho PH, Cruz R, Kappann JI, Júnior LAT. Consumo de drogas psicoativas por adolescentes escolares de Assis, SP. Rev Saúde Pública 2004;38(1): 130-2.

12. Andrade SSCDA, Yokota RTDC, Bandeira SNN, Silva MMAD, Araújo WND, Mascarenhas MDM, Malta DC. Relação entre violência física, consumo de álcool e outras drogas e bullying entre adolescentes escolares brasileiros. Cad Saúde Pública 2012;28(9):1725-36.

13. Zeitoune RCG, Santos FV, Silveira HS, Domingos AM, Maia ACO. Conhecimento de adolescentes sobre drogas lícitas e ilícitas: uma contribuição para a enfermagem comunitária. Esc Anna Nery Rev Enferm 2012;16(1):57-63.

14. Precioso J. Educação para a saúde na escola Um direito dos alunos que urge satisfazer. O Professor 2004;85:17-24.

15. Malta DC, Sardinha LMV, Mendes I, Barreto SM, Giatti L, Castro IRR, et al. Prevalência de fatores de risco e proteção de doenças crônicas não transmissíveis em adolescentes: resultados da Pesquisa Nacional de Saúde do Escolar (PeNSE), Brasil, 2009. Ciênc Saúde Coletiva 2010;15(2):3009-19.

16. Figueiredo TAM, Machado VLT, Abreu MMS. A saúde na escola: um breve resgate histórico. Ciênc Saúde Coletiva 2010;15(2):397-402. 